\title{
Genesis
}

Manuscrits - Recherche - Invention

$50 \mid 2020$

Aragon

\section{Le séduisant roman de la genèse des romans}

\section{Daniel Bougnoux}

\section{OpenEdition}

\section{Journals}

\section{Édition électronique}

URL : https://journals.openedition.org/genesis/5173

DOI : 10.4000/genesis.5173

ISSN : 2268-1590

\section{Éditeur :}

Presses universitaires de Paris Sorbonne (PUPS), Société internationale de génétique artistique littéraire et scientifique (SIGALES)

\section{Édition imprimée}

Date de publication : 15 juillet 2020

Pagination : 33-37

ISBN : 979-10-231-0679-4

ISSN : 1167-5101

\section{Référence électronique}

Daniel Bougnoux, «Le séduisant roman de la genèse des romans », Genesis [En ligne], 50 | 2020, mis en ligne le 15 juillet 2021, consulté le 12 janvier 2023. URL : http://journals.openedition.org/genesis/ 5173 ; DOI : https://doi.org/10.4000/genesis.5173

Ce document a été généré automatiquement le 12 janvier 2023.

Tous droits réservés 


\title{
Le séduisant roman de la genèse des romans
}

\author{
Daniel Bougnoux
}

1 Faut-il voir, à la suite de Louis Hay brandissant, lors d'une assemblée de l'ITEM, son exemplaire de Je n'ai jamais appris à écrire ou les incipit (1969), orné d'une flatteuse dédicace de son auteur au promoteur de la critique génétique, un manifeste bienvenu d'Aragon inaugurant par ce titre un courant d'études?

2 Ou mieux, avec le geste, quelques années plus tard (mai 1977), de déposer solennellement au CNRS la plupart de ses manuscrits disponibles, ainsi mis à la disposition des chercheurs?

3 J'aurai moi-même, éditant les cinq volumes de ses CEuvres romanesques complètes dans la Bibliothèque de la Pléiade, beaucoup consulté ce fonds d'archives ; son examen ne m'a pas tellement éclairé sur les sentiers aragoniens de la création (pour reprendre le titre de la collection lancée par Albert Skira et Gaëtan Picon). Je revois en particulier, dans le manuscrit de La Mise à mort, une demie-page entièrement noircie d'encre pour mieux la raturer, ou tel titre impitoyablement caviardé, sans aucune sous-lecture possible: quand Aragon se corrigeait, je comprends par ces exemples qu'il ne laissait aucune chance à la version première, de sorte qu'on peut supposer qu'il aura détruit ou fait disparaître quantité d'avant-textes, au lieu de les joindre obligeamment aux cartons destinés au CNRS... La déférence envers les généticiens n'était pas son fort! Ni, en général, le souci de s'expliquer.

4 Très tôt pourtant, dès Anicet ou le panorama (1921), les textes d'Aragon se trouvent parsemés de remarques métalinguistiques, comme s'il anticipait déjà la question qui dominera Banche ou l'oubli (1967), de savoir « comment cela marche une tête ». Ou une plume... Notre auteur s'interroge ainsi, dès son premier roman, sur la survenue automatique de certaines images, ou d'enchaînements verbaux qui s'imposent à sa pensée ; l'absence de préméditation, la surprise ou le hasard des "coups » semblent conduire à son insu sa main, et dans cette mesure, participent à une relative décapitation du sujet (concentrée dans la figure de l'acéphale à la fin du Paysan de Paris). La conception convenue d'un auteur dominant de sa hauteur l'acte de création s'en 
trouve ébranlée; l'écriture (au moins dans le cas d'Aragon) n'est pas quelque chose qu'on dirige, qu'on prémédite ni qu'on s'approprie jamais entièrement.

Or cette autonomie ou cet automatisme relatifs du processus créatif s'étaient trouvés dénudés au printemps 1919 avec l'invention de l'écriture automatique par Breton et Soupault, lancés dans l'aventure des Champs magnétiques alors qu'Aragon stationnait encore dans la Sarre; l'affaire ne pouvait que susciter sa curiosité, autant qu'une certaine jalousie. Cet automatisme définira de fait, dans le Manifeste surréaliste de 1924, le principe même du mouvement ainsi baptisé: «Automatisme psychique pur. [...] Dictée de la pensée, en l'absence de tout contrôle conscient ».

6 À l'époque de cette définition pourtant, l'écriture automatique devenue répétitive aux yeux de Breton semblait n'entraîner qu'une suite de déconvenues; et Aragon lui-même n'y aura guère sacrifié, sous sa forme stricte. Un texte de lui paraitra en décembre 1926, dans La Révolution surréaliste, "Moi l'abeille j'étais chevelure", sous ce label d'une stricte orthodoxie, mais un adieu à cette pratique désormais « classique » s'y dessine et Traité $d u$ style, un an plus tard, en dressera rigoureusement le bilan.

7 Aragon y proteste hautement contre ceux qui n'y voient qu'un élargissement du vers libre, et qui croient avoir "trouvé le système »: on se lâche, on barbote dans la nouvelle démocratie du génie, «[...] tout le monde, après avoir dit un poème dada, rien de plus simple, tenez saut à charbon bonbons confitures, s'écrie le surréalisme j'en suis : les cuisses des horizontales obsolètes... (car ces acrobates trouvent le surréalisme un peu bordel)». Et d'enfoncer le clou: "Si vous écrivez, suivant une méthode surréaliste, de tristes imbécillités, ce sont de tristes imbécillités. Sans excuses ».

8 Au moment (1927) où le mouvement risque d'être rattrapé par les divers démons de la mode, des gogos, de l'académisme ou de la paresse intellectuelle, Aragon s'efforce dans ces pages railleuses de rendre au surréalisme, c'est-à-dire à l'automatisme, sa rigueur :

Le surréalisme est l'inspiration reconnue, acceptée, et pratiquée. Non plus comme une visitation inexplicable, mais comme une faculté qui s'exerce. Normalement limitée par la fatigue. D'une ampleur variable suivant les forces individuelles. Et dont les résultats sont d'un intérêt inégal. [...] L'homme qui tient la plume ignore ce qu'il va écrire, ce qu'il écrit [...]. Rigueur inévitable. Le sens se forme en dehors de vous.

Dans un texte peu lu de 1937 repris dans Le Mentir-vrai, « Un roman commence sous vos yeux », Aragon a voulu revenir sur cette « visitation inexplicable » et nous en proposer une démystification. L'ardent réaliste qu'il est devenu n'a rien oublié alors de sa formation surréaliste, qu'il définit à présent comme une tentative particulièrement radicale de réduction du mécanisme de l'inspiration poétique. Mais les surréalistes reculèrent devant leur propre projet, et ils remontèrent à leur profit la mécanique qu'ils avaient si bien démontée. Aragon pour sa part refuse de nous mystifier davantage ; la poésie, l'inspiration, le "génie » ne doivent pas devenir l'asile de l'ignorance, ni couvrir une abdication critique. Ce n'est donc pas lui, le converti au réalisme, mais eux qui ont trahi cet idéal analytique, en refusant de tirer au clair les mécanismes de l'inspiration. Ce que ce court texte se propose enfin de dévoiler. Ces mécanismes ne sont pas seulement psychologiques mais sociaux; le réalisme prolonge le surréalisme en l'étendant vers des cercles plus larges d'explication.

10 Ce sur quoi notre auteur passe à la pratique en forgeant pour nous, pédagogiquement, l'exemple d'un incipit de roman : «Claudine, avec son air de ne pas y toucher, faisait 
dans la petite ville l'effet d'un arc-en-ciel dans la pampa... ${ }^{1}$ " En quoi cette phrase dictée, ou de réveil comme disait aussi Breton, a-t-elle valeur explicative?

Beaucoup plus tard, dans Je n'ai jamais appris à écrire ou les incipit (Skira, 1969) dont ce texte de 1937 constitue une anticipation remarquable, Aragon nous « expliquera » la valeur génératrice de telles phrases : c'est en les entendant, ou en les recopiant avec incrédulité, qu'il écrit une seconde phrase pour justifier la première, une troisième au secours de la seconde et que de fil en aiguille... « Je n'ai jamais écrit mes romans, je les ai lus. [...] J'ai été mené par une rencontre de mots, ou de sons, la nécessité d'une allitération, la logique de l'illogisme, la légitimation après coup d'un heurt des mots » déclare-t-il en 1969, comparant cet automatisme à la carambole au billard. De fait, cette thèse renversante semble la résurgence du filon détecté d'abord par le couple BretonSoupault; si l'on en croit Aragon, lui-même aurait écrit ses romans "automatiquement» - une thèse qui, étant donné leur degré d'érudition ou de documentation (songeons à La Semaine sainte), prête à sourire !

Il est plausible que certains incipit lui fussent « dictés », et que l'affirmation de 1969 ait une vérité relative : Le Con d'Irène déjà (1928) nous faisait assister à certaines scènes dont l'emportement maldororien plaide en faveur de cette curieuse méthode. Et on y lit déjà la thèse selon laquelle l'écriture, bien loin de succéder à la pensée, la précède... Aragon a tellement écrit (plusieurs pages chaque jour si l'on prend la mesure de son œuvre) qu'il lui fallait bien aller vite, sinon plume courante (ce que certains témoignages aussi attestent).

Le dessaisissement, la surprise, le choc ou la recherche d'un certain vertige conduisent donc cette esthétique paradoxale d'Aragon, qui part de la table rase ou recommence chaque fois à zéro, qui cherche (à voir, à connaître, à ressentir) au cœur même ou au ras de ce qu'il écrit. Mais l'hypothèse même de l'incipit comme levier de la création, en concentrant l'irruption du génie ou de la grâce au commencement d'écrire, ne peut nullement servir à démystifier cette inspiration à laquelle il prétend tordre le cou. Déplacée, chassée par la porte, elle rentre par la fenêtre. Il faut beaucoup de naïveté pour voir dans ce livre de 1969, qui lui fait un pied-de-nez moqueur, un fondement de la critique dite depuis " génétique ». Les Incipit ne nous explique à aucun degré la création romanesque de leur auteur; le magicien, en feignant de nous expliquer son tour, nous remystifie toujours davantage.

14 "J'ai aussi décrit des phrases, quand on croyait que je les écrivais » énonce, assez énigmatiquement, la « Préface à l'édition du Libertinage de $1924^{2}$ ». Le roman serait-il la description continuée, pleinement étendue de son incipit? Celui-ci est parfois donné comme phrase entendue, c'est le cas notamment des Voyageurs de l'impériale, mais aussi du Roman inachevé, ou du Fou d'Elsa. La tardive théorie des Incipit, annoncée de mille façons depuis les années vingt, introduit le rapport texte-lecteur au cœur de l'écriture : écrire, ce serait lire, étayer l'une sur l'autre des phrases successives qui se développent en s'interprétant rétrospectivement. L'incipit pose, ou colle, une phrase donnée (dictée), énigmatique, dont la suite du roman rémunère (réduit, justifie) peu à peu l'étrangeté.

"Voici le temps enfin qu'il faut que je m'explique » : ce titre, par antiphrase sans doute ironiquement emprunté à Racine pour cautionner la préface d'Aurélien, déjoue d'avance toute explication du roman princeps d'Aragon: son auteur n'a aucune intention de retourner ses cartes, de révéler ses pilotis ni, en général, d'en dire trop sur ses sources ou sentiers; une bonne part du charme d'Aurélien tient au contraire à la stratégie de l'esquive, et du voilé-montré, qui nimbent méticuleusement le jeu des personnages. Au 
sens étymologique en revanche, il est vrai que l'incipit est un pli, qui fait énigme, et que l'écriture romanesque progresse en dépliant cette énigme ou ce pli, par une seconde phrase (second pli) qui elle-même, à son tour, en appelle une troisième, etc. La démarche n'est pas théorique-explicative, mais explicitante, le roman s'auto-déplie, le lire c'est assister à un déploiement - sans aucune visée de transparence finale. Cheminant de pli en pli, nous aurons comme Michaux vécu "dans les plis ${ }^{3}$ », lesquels constituent bien le lieu (paradoxal) de l'étrange vie des livres.

16 Si la chaîne des phrases ou des signifiants s'avère interminable, c'est que d'une certaine manière il n'y a que du métalangage (position explicitée avec l'émergence de la linguistique dans Blanche ou l'oubli de 1967), un mouvement perpétuel de reprises et d'interprétations: Aragon entretiendrait ou rejouerait vis-à-vis de son œuvre une relation de lecteur à texte, dénudée dans Le Mentir-vrai puis Les Incipit.

Le surréalisme aura donc été conduit par lui comme une expérience de pensée, «le plus grand effort que je connaisse de réduction [du mécanisme poétique] », résume-t-il luimême après coup dans La Souris rouge $(1933)^{4}$. D'où les accusations de certains de ses amis, lui reprochant de n'avoir été qu'un surréaliste « pour voir». Si «l'automatisme psychique pur, dictée de la pensée [...] » définit le surréalisme, il est patent qu'Aragon pratiqua assez peu ce lâchez-tout, pas plus qu'à l'époque des sommeils - auxquels excellaient Crevel ou Desnos - il ne consentit à s'endormir. Ses textes s'efforcent de rapatrier dans la lumière de la veille les sortilèges de l'ombre, et de réussir la difficile synthèse de l'improvisation sauvage et de la culture, de la frénésie et de la raison.

Le récit du Paysan de Paris portait à l'extrême ce défi - qui inspirait déjà plusieurs contes du Libertinage, notamment « Paris la nuit »- de marier sous le faux jour des verrières du passage, ou dans la nuit des Buttes-Chaumont, l'observation la plus aiguë avec les projections du monde intérieur, et de développer du même pas le lyrisme et la conscience critique, la fuite des idées et leur suite dans le raisonnement. Philosophe, Aragon voudrait effectuer la synthèse du romantisme et de la philosophie des Lumières, mais il sait tout ce que ces lumières peuvent avoir de mortel pour les jeux du désir et de l'imagination. Un même texte, Le Con d'Irène, combine ainsi les deux postures en s'ouvrant sur les hurlements d'une conscience arrachée au sommeil, «Ne me réveillez pas, nom de Dieu, salauds, ne me réveillez pas ", pour se refermer sur des considérations sagement métalinguistiques telles que: "Supposez que tout ce que je dis a un caractère plutôt scientifique ».

19 Son génie fut de se mouvoir entre ces deux pôles d'une folie recherchée ou frôlée, et d'une critique attentive aux mécanismes de cette folie; et de réussir à marier parfois les deux postures jusque dans la même page.

"Je ne suis pas un magicien, cette constatation ne va pas sans tristesse..$^{5}$ » Au beau temps du réalisme socialiste, le texte pédagogique et clinique de «Un roman commence sous vos yeux" se souvient de la formation du médecin; partisan d'une raison expérimentale, Aragon avait lui-même critiqué la façon dont le surréalisme, " extrême pointe de l'idéalisme artistique ${ }^{6} "$, après avoir démonté mieux que personne les mécanismes de la création poétique, l'avait ensuite remonté pour faire tourner cette mécanique à son profit. Trop intelligent pour jouer au magicien ou au mage, il voudrait avec ce texte et quelques autres nous expliquer le génie. Mais tout se passe comme plus tard dans Les Incipit, ce séduisant roman de la genèse des romans : au moment où le prestidigitateur prétend nous dévoiler comment ça marche, il ne peut s'empêcher 
d'exécuter un autre tour qui de plus belle nous mystifie. La «théorie » des Incipit ou de la phrase de réveil, bien loin de rien expliquer, fait rebondir l'énigme ou le mystère.

\section{NOTES}

1. Louis Aragon, CEuvres romanesques complètes II, D. Bougnoux (dir.), Paris, Gallimard, coll. "Bibliothèque de la Pléiade ", 2007, p. 485.

2. Louis Aragon, Euvres romanesques complètes I, D. Bougnoux (dir.), Paris, Gallimard, coll. «Bibliothèque de la Pléiade », 2007, p. 281.

3. Henri Michaux, La Vie dans les plis, Paris, Gallimard.

4. Louis Aragon, "La Souris rouge ", dans l'édition en quinze volumes du Livre-Club Diderot, vol. V, p. 383.

5. Louis Aragon, Le Con d'Irène, dans Euvres romanesques complètes I, op. cit., p. 447.

6. Louis Aragon, Euvres romanesques complètes II, op. cit., p. 485.

\section{RÉSUMÉS}

Faut-il croire Aragon quand il expose, dans Je n'ai jamais appris à écrire ou les incipit (1969), que ses romans sont nés d'une phrase de réveil posée comme un collage, qui exige pour son développement une deuxième phrase, laquelle en appelle une troisième, etc. ? « Mes romans je les ai lus... » Cette thèse renversante voudrait renouer avec l'écriture automatique, inventée par Breton et Soupault en 1919, qui servira de définition canonique au surréalisme. L'automatisme entre assurément dans le processus d'écriture pratiqué par Aragon, qui parsème ses livres de remarques métalinguistiques touchant le surgissement d'une image, ou d'un vocable, et qui s'inquiète de savoir à partir de là " comment cela marche une tête ». Ou une plume. Le génie d'Aragon semble ainsi tiraillé entre les deux extrêmes d'une décapitation ou d'une folie recherchée, assumée au fil des phrases, mais doublée d'une vigilance critique et du souci de toujours expliquer les mécanismes de la création, chez lui comme chez les autres.

Can we believe Aragon when he tells us, in I never learnt to write or the incipit (1969), that his novels started from an arousing sentence caught from nowhere, a collage asking to be developped by another sentence, which in turn calls for a third, and so on? "My novels, I have read them..." This smashing thesis seems to be an attempt to reconnect with the automatic writing invented by Breton and Soupault in 1919, which gave surrealism its definition. Automatism is a clear componant of Aragon's process of writing, whose books are sparsed with metalinguistic notices questioning the outbreak of an image, or a noun, asking "how does a head work". Or a pen. Aragon's genius seems to be stretched between two extremes, a decapitation or a madness. A madness cultivated all along his narratives, but folded into the standpoint of a critic, always eager to explain the mecanisms of creation, for himself as well as for the others. 
Sollen wir Aragon glauben, wenn er in Je n'ai jamais appris à écrire ou les incipit (1969) erklärt, dass seine Romane aus einem collageartig angelegten Satz entstanden sind, den er beim Aufwachen notierte und der für seine Entwicklung einen zweiten Satz, welcher wiederum einen dritten usw. erfordert? „Mes romans je les ai lus...“ Diese verblüffende These möchte die sogenannte „écriture automatique“, die1919 von Breton und Soupault erfunden wurde, wiederbeleben, welche nachher als kanonische Definition des Surrealismus dienen wird. Der Automatismus ist sicherlich Teil des Schreibprozesses von Aragon, der seine Bücher mit metalinguistischen Bemerkungen über die Entstehung eines Bildes oder eines Wortes bestreut und der von dort aus mit Sorgen fragt, „wie ein Kopf funktioniert“. Oder eine Feder. Das Genie Aragons scheint also zwischen zwei Extremen hin- und hergerissen zu sein, nämlich zwischen einer Enthauptung oder eines Wahnsinns einerseits, die im Laufe der Sätze gesucht, ja für sich angenommen werden, und aber auch andererseits einer kritischen Wachsamkeit und dem Anliegen, die Mechanismen der Schöpfung bei ihm wie bei anderen immer zu erklären.

¿Se le puede creer a Aragon cuando explica, en Je n'ai jamais appris à écrire ou les incipit (1969), que sus novelas han nacido de una frase desencadenante articulada como un collage, que requiere para su desarrollo una segunda frase, que a su vez exige una tercera, etc.? "Yo he leído mis novelas..." Esta tesis impactante intenta restablecer el vínculo con la escritura automática, inventada por Breton y Soupault en 1919, que servirá como definición canónica al surrealismo. El automatismo participa indudablemente en el proceso de escritura que practica Aragon, quien esparce en sus libros observaciones metalingüísticas relativas al surgimiento de una imagen o de un vocablo, a partir del cual intenta entender "cómo funciona la cabeza". o la pluma. De este modo, el genio de Aragon parece debatirse entre los dos extremos de una decapitación o de una locura buscada, asumida a lo largo de las frases, pero acompañada por una vigilancia crítica y la voluntad de explicar permanentemente los mecanismos de la creación, tanto en su propia obra como en la de otros.

Deve-se acreditar em Aragon quando ele expõe, em Nunca aprendi a escrever ou os incipit (1969), que seus romances nasceram de uma frase qualquer, tirada do nada, posta como uma colagem que exige para se desenvolver uma segunda frase, que por sua vez, pede uma terceira, etc.? «Meus romances, eu os li...» Esta tese surpreendente parece querer reatar com a escrita automática, criada por Breton e Soupault, em 1919, que servirá de definição canônica ao surrealismo. $\mathrm{O}$ automatismo faz parte certamente do processo de escrita praticado por Aragon, que espalha em seus livros notas metalinguísticas que permitem o surgimento de uma imagem, ou de um vocábulo, questionando a partir daí « como funciona uma cabeça ». Ou uma caneta. A geniosidade de Aragon parece assim estar dividida entre os dois extremos de uma decapitação ou de uma loucura procurada, assumida ao longo das frases, mas acompanhada de uma vigilância crítica e da problemática de sempre explicar os mecanismos da criação, tanto da sua obra como das dos outros.

Dobbiamo credere ad Aragon quando ci confessa, in Je n'ai jamais appris à écrire ou les incipit (1969), che i suoi romanzi sono nati da una frase al risveglio, posata lì come un collage, che esige per il suo sviluppo una seconda frase, la quale, a sua volta, ne richiama una terza, eccetera ? "I miei romanzi, li ho letti..." Questa tesi stupefacente vorrebbe riallacciarsi alla scrittura automatica, inventata da Breton e Soupault nel 1919, che diventerà la definizione canonica del surrealismo. L'automatismo fa indubbiamente parte del procedimento di scrittura di Aragon, che dissemina i suoi libri di osservazioni metalinguistiche sulla nascita di un'immagine o di un vocabolo e si preoccupa, a partire da ciò, di sapere "come funziona una testa". O una penna. Il genio di Aragon sembra così combattuto tra i due estremi di una decapitazione o di una follia ricercata, accettata frase dopo frase, ma accompagnata da una vigilanza critica e dalla preoccupazione di spiegare sempre i meccanismi della creazione, la sua come quella degli altri. 
INDEX

Mots-clés : mécanismes de la création, écriture automatique, genèse littéraire, Aragon Louis, génie, Je n'ai jamais appris à écrire ou les incipit

\section{AUTEUR}

\section{DANIEL BOUGNOUX}

DANIEL BOUGNOUX, philosophe, ancien élève de l'École Normale Supérieure (Ulm), est professeur émérite de l'Université des Alpes à Grenoble, où il a successivement enseigné la littérature, puis les sciences de l'information-communication. Lié avec Régis Debray, il a participé à la fondation des Cahiers de médiologie (Gallimard) devenus la revue Médium. Auteur d'une vingtaine d'ouvrages, il tient aussi régulièrement le blog «Le Randonneur », hébergé par La Croix. De 1997 à 2012, il a dirigé l'édition en cinq volumes des Euvres romanesques complètes d'Aragon dans la Bibliothèque de la Pléiade ; et en août 2018, avec Luc Vigier, le colloque « Aragon vivant », à Cerisy-la-Salle. 